\title{
A Multi-Temporal Optimal Power Flow for Managing Storage and Demand Flexibility in LV Networks
}

\author{
H. M. Costa, J. Sumaili, A. G. Madureira and C. Gouveia \\ Centre for Power and Energy Systems (CPES) \\ INESC Technology and Science (INESC TEC) \\ Porto, Portugal \\ jean.sumaili@inesctec.pt, helder.m.costa@inesctec.pt, andre.g.madureira@inesctec.pt, clara.s.gouveia@inesctec.pt
}

\begin{abstract}
This paper presents an algorithm developed for the optimization of Low Voltage (LV) grids that takes advantage of Distributed Energy Resources (DER) such as storage devices and flexible loads. The proposed approach is based on a multitemporal Optimal Power Flow (OPF) algorithm that feeds from forecasting tools for load and renewable generation, which means the optimization model looks at a 24-hours horizon with hourly resolution. Specific constraints to the OPF are added to adequately model storage devices, namely their State-of-Charge (SOC) limits as well as their charging and discharging efficiencies. Moreover, a full three-phase model was built due to the unbalanced nature of $L V$ grids motivated by the presence of single-phase load and generation. The algorithm developed has been extensively tested through simulation using a real LV Portuguese network data to illustrate the performance of the algorithm in different scenarios with good results.
\end{abstract}

Index Terms-Optimal Power Flow, Multi-Temporal, Voltage Control, Storage Devices, Demand Flexibility, Unbalanced Network.

\section{INTRODUCTION}

The connection of Distributed Generation (DG), especially based on Renewable Energy Sources (RES), is becoming a challenge for distribution system operation due to the variable nature of the power produced [1]. In this context, storage is being regarded as a potential solution to mitigate the impacts of this variability [2]. Storage systems may enable smoothing the generation profile of these units while avoiding the injection of power into the distribution grid, which is often a cause of constraints to grid operation. In particular, voltage problems or congestions on lines or transformers are critical issues in the case of LV distribution grids.

Therefore, the possibility of monitoring and the control capability of LV network assets is becoming increasingly important [3]. In particular, the integration of microgeneration in $\mathrm{LV}$ networks associated to the need of improving power quality and continuity of service requires not only the integration of flexible resources such as storage systems, but also the development and implementation of specific tools designed to support grid operation, which will enable

The research leading to these results has received funding from the European Union's Horizon 2020 - The EU Framework Programme for Research and Innovation 2014-2020, under grant agreement No. 645963. identifying and solving technical problems that may occur at the distribution grid level [4].

The connection of DER to the LV grid is foreseen to increase substantially in the future, including not only storage devices owned by the Distribution System Operator (DSO) but also other type of resources of domestic clients such as controllable loads, small domestic storage systems, small photovoltaic (PV) microgeneration units or electric water heaters with continuous power regulation [5]. Therefore, managing the flexibility of consumers' loads by managing their resources, based on a remuneration mechanism defined in a market framework or through bilateral contracts, is crucial in order to take full advantage of the capability of the presence of these resources to support network operation.

The main objective of the proposed approach is to optimize LV network operation while minimizing the deviation between actual and expected net load profile. It makes use of storage devices connected to the secondary substation, or directly in LV distribution feeders, as well as demand response actions. The algorithm developed optimizes the storage and controllable loads operating strategy for a pre-defined time horizon (e.g., next hours, day) in order to minimize the power losses, respecting the technical constraints of the network (namely in terms of voltage profiles) and considering the future states of the LV grid.

This tool has a preventive nature by taking advantage of forecast data to anticipate technical problems that may arise during operation, namely in terms of voltage profiles [6]. The final output consists of a set of set-points that represent the operating strategy of the storage devices and controllable loads for the next hours/day. In this case, the controllable loads are assumed to be managed through a Home Energy Management System (HEMS) that allows controlling the flexible resources of LV clients regardless of how many different resources one specific client may have.

The formulation of the multi-temporal OPF algorithm is described in section II. The functional specification of the tool is presented in section III. The most relevant results obtained are included in section IV and, finally, in section $\mathrm{V}$ the main conclusions are drawn. 


\section{MATHEMATICAL FORMULATION}

As previously explained, the algorithm defines the optimal operation strategy for a full day, considering time steps of one hour. In order to consider all the hours of a day in a single optimization problem, the network is replicated 24 times and additional constraints are taken into account in order to model the inter-temporal restrictions introduced by the storage devices.

Consequently, the equivalent three-phase network will have a total of $N=3 \times 24 \times n$ buses, where $n$ is the number of buses in the "original" network. Similarly, the total number of "virtual generators" (i.e. resources that can be controlled in terms of $\mathrm{P}$ and Q) will be equal to $N_{g}=3 \times 24 \times$ $\left(n_{g}+2 \times n_{s}+2 \times n_{f}\right)$, where $n_{g}$ is the number of generators in the real network, $n_{f}$ the number of flexible loads and $n_{s}$, the number of storage devices.

The resulting variable vector includes the state and control variables for the 24 hours of the day, as in (1).

$$
\begin{aligned}
X & =(\Theta, \mathrm{V}, \mathrm{P}, \mathrm{Q}) \\
\Theta & =\left\{\theta_{1}^{1}, \ldots, \theta_{3 n}^{1}, \ldots, \theta_{1}^{24}, \ldots, \theta_{3 n}^{24}\right\} \\
\mathrm{V} & =\left\{\left|V_{1}^{1}\right|, \ldots,\left|V_{3 n}^{1}\right|, \ldots,\left|V_{1}^{24}\right|, \ldots,\left|V_{3 n}^{24}\right|\right\} \\
\mathrm{P} & =\left\{P g_{1}^{1}, \ldots, P g_{N_{g} / 3}^{1}, \ldots, P g_{1}^{24}, \ldots, P g_{N_{g} / 3}^{24}\right\} \\
\mathrm{Q} & =\left\{Q g_{1}^{1}, \ldots, Q g_{N_{g} / 3}^{1}, \ldots, Q g_{1}^{24}, \ldots, Q g_{N_{g} / 3}^{24}\right\}
\end{aligned}
$$

Where,

$$
\begin{aligned}
& X \quad-\quad \text { Variable vector } \\
& \Theta \quad-\quad \text { Vector with of voltage angles } \\
& \mathrm{P} \quad-\quad \text { Vector with the resulting active power set- } \\
& \text { points of "virtual" generators } \\
& \text { Q - - Vector with the resulting reactive power set- } \\
& \text { points "virtual" generators } \\
& \theta_{n}^{\mathrm{i}} \quad-\quad \text { Voltage angle in bus } n \text { for the } i^{\text {th }} \text { hour } \\
& P g_{n_{g}}^{\mathrm{i}}, \quad-\quad \text { Active and reactive power set-point for } \\
& Q g_{n_{g}}^{24} \quad \text { respectively }
\end{aligned}
$$

The Interior Point Method was chosen for solving the OPF problem formulated here [7]-[9].

\section{A. Control Variables}

Regarding the control variables, the OPF algorithm implemented considers the following:

- Active $(P)$ and reactive power $(Q)$ of storage unit inverter(s);

- Active power of controllable loads (domestic client flexibility activated through the HEMS)

- Voltage magnitude of the reference node $\left|V_{\text {ref }}\right|$.

In this case, different weights are given for each of these control variables. Therefore, it was considered that storage units should always be used before any flexibility from loads is required to be activated. This was modelled by implementing different costs for the use of these resources (a higher cost for using flexibility from loads than for using storage).

\section{B. Objective Function}

The objective function considered was global loss minimization as shown in (6) where $P_{j, t}^{i n j}$ and $\varepsilon_{j}^{i n j}$ are the injected active power of the storage device $j$ during period $t$ and its discharge efficiency, respectively, while $\Delta P_{k, t}^{\text {down }}$ and $\Delta P_{k, t}^{u p}$ are the decrease and the increase of flexible load $k$, respectively.

$$
\sum_{t=1}^{24}\left(\sum_{i=1}^{n_{g}} P_{i, t}^{g}+\sum_{j=1}^{n_{s}} \frac{P_{j, t}^{i n j}}{\varepsilon_{j}^{i n j}}+\sum_{k=1}^{n_{f}} a_{k}\left(\Delta P_{k, t}^{\text {down }}+\Delta P_{k, t}^{u p}\right)\right)
$$

$a_{k} \gg 1$ increases the weight of load flexible in order to define a merit for the use of flexibility allowing its activation only when there is no other alternative without network constraint violation.

\section{Constraints}

The decision variables referred are limited by constraints, presented in (7). For example, when DG is available, the active and reactive power set-points are defined according to the generator' limits. Also, the OPF solution will only be valid if network limits are respected, namely the voltage profile must be within specified limits and the line power limits must also be respected.

$$
\underline{X} \leq X \leq \bar{X}
$$

The equality constraints shown in (8) correspond to the full $\mathrm{AC}$ power flow equations for a three-phase system.

$$
\begin{array}{ll}
\left|S_{f, j}\right|^{2} \leq S_{b}^{\text {rate }}{ }^{2}, & j \in\{1, \ldots, b\} \\
\left|S_{t, j}\right|^{2} \leq S_{b}^{\text {rate }}{ }^{2}, & j \in\{1, \ldots, b\}
\end{array}
$$

Where,

$$
\begin{array}{ll}
\left|S_{f, j}\right|,\left|S_{t, j}\right|- & \begin{array}{l}
\text { Apparent power flowing in each branch } \mathrm{j} \\
\text { from the sending to the ending node, } \\
\text { respectively }
\end{array} \\
S_{b}^{\text {rate }} & -\quad \text { Rated power of the branch } \\
b \quad-\quad \begin{array}{l}
\text { Total number of branches of the } \\
\text { distribution network }
\end{array}
\end{array}
$$

The equality constraints imposed by the power balance of each node of the network and for each period are shown in (10) and (11). Here, the indices of the hours and phases have been omitted for readability purposes only.

$$
\begin{array}{r}
\left|V_{k}\right| \sum_{m=1}^{N}\left|V_{m}^{f}\right|\left(G_{k m} \cos \left(\theta_{k m}\right)+B_{k m} \sin \left(\theta_{k m}\right)\right) \\
+P_{g k}-P_{l k}=0, k \in\{1, \ldots, N\}
\end{array}
$$




$$
\begin{array}{r}
\left|V_{k}\right| \sum_{m=1}^{N}\left|V_{m}^{f}\right|\left(G_{k m} \sin \left(\theta_{k m}\right)-B_{k m}^{f} \cos \left(\theta_{k m}\right)\right) \\
+Q_{g k}-Q_{l k}=0, k \in\{1, \ldots, N\}
\end{array}
$$

\begin{tabular}{|c|c|}
\hline$\left|V_{k}\right|,\left|V_{m}\right|$ & - Voltage magnitude of bus $k$ and bus $m$, \\
\hline$\theta_{k m}$ & $\begin{array}{l}\text { - Voltage angle difference between bus } k \\
\text { and bus } m\end{array}$ \\
\hline$G_{k m}, B_{k m}$ & $\begin{array}{l}\text { Real and imaginary parts of the elements } \\
\text { of the } 3 n \times 3 n \text { admittance matrix, } \\
\text { respectively }\end{array}$ \\
\hline$P_{g k}$ & $\begin{array}{l}\text { - Active power of generator connected to } \\
\text { bus } k\end{array}$ \\
\hline$l_{l k}$ & Active power consumed in bus $k$ \\
\hline$Q_{a k}$ & $\begin{array}{l}\text { - Reactive power of generator connected to } \\
\text { bus } k\end{array}$ \\
\hline & Reactive power consumed in bus $k$ \\
\hline & Total number of buses of the network \\
\hline
\end{tabular}

Where,

As previously mentioned, the main feature of this algorithm is the integration of the storage unit(s) in the OPF algorithm, which originate the multi-period constraints related to the operation of the storage device(s) [10].

From an energy balance point of view, the efficiency of the storage process needs to be considered. This introduces a discontinuity in the storage model and in the corresponding restrictions. Figure 1. illustrates the model implemented for a single-phase storage device. It includes two different elements with continuous behavior: a load during the charging period, a generator for power supply. The SOC takes into account the losses during the charging and the injecting periods of the battery.

$P_{t}^{c h}$ is the load power representing the storage behavior during the charging period $t$ and the power of corresponding negative generator is then $-P_{t}^{c h}$;

$P_{t}^{i n j}$ is the storage power injection during the period $t$;

$\left(1-\varepsilon^{c h}\right) . P_{t}^{c h}$ and $\left(1-\varepsilon^{i n j}\right) . P_{t}^{i n j} \quad$ represent the storage charging and discharging losses, respectively.

The modelling approach followed requires the definition of new restrictions to the OPF problem, corresponding to the state of the charge for each storage device.

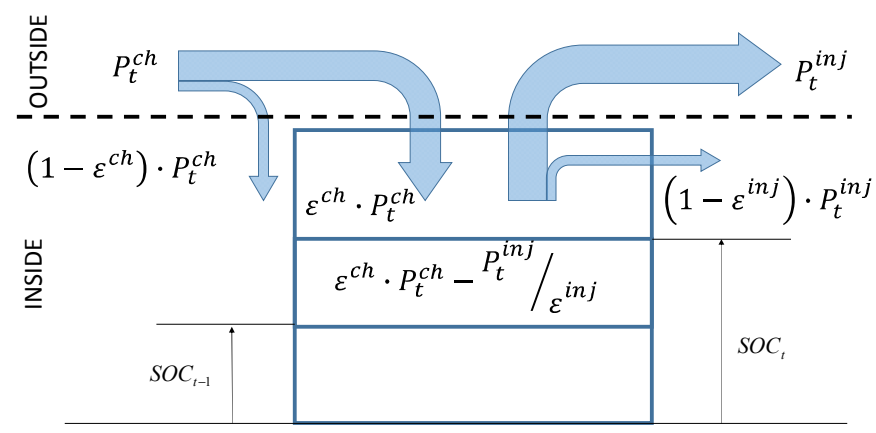

Figure 1. Storage Device Model
The $S O C$ of a single storage in each hour can be calculated as in (9) where $j \in\left\{1, \ldots, n_{s}\right\}$.

$$
S O C_{t, j}=S O C_{t-1, j}+\varepsilon_{j}^{c h} \cdot P_{t, j}^{c h}-\frac{P_{t, j}^{i n j}}{\varepsilon_{j}^{i n j}}
$$

The limits for the $S O C$ were also considered for each storage device as shown in (10) where $\overline{S O C}_{j}$, the upper limit, is the storage capacity of the storage device and $S O C_{t, j}$, the lower limit. The lower limit of the storage SOC is defined for each hour according to the required backup capacity that needs to be ensured for purposes of quality and continuity of service, namely considering the load forecasting for the next hour(s).

$$
\underline{S O C}_{t, j} \leq S O C_{t, j} \leq \overline{S O C}_{j}
$$

In case of three-phase storage device, the $S O C$ will consider the contribution of the three phases. If the injection (or consumption) of each phase cannot be controlled separately, the optimization problem should include more restrictions that impose the three phases to have the same behavior as shown in in equation (14) and (15)

$$
\begin{aligned}
& P_{t, j}^{a}=P_{t, j}^{b}=P_{t, j}^{c} \\
& Q_{t, j}^{a}=Q_{t, j}^{b}=Q_{t, j}^{c}
\end{aligned}
$$

where the superscripts $a, b$, and $c$ are related to each of the three phases.

\section{Optimization TOOL STRUCTURE AND SETUP}

As represented in Figure 2. the tool will run day-ahead (d1) in order to define the LV optimal strategy for the storage units and other DER (namely demand flexibility of domestic clients via the HEMS) and identify potential technical restrictions that cannot be solved by the algorithm. Then, during the day (d), before sending the set-points directly to the different DER the tool will validate the set-points defined in the previous day based on the current state of LV network. This will avoid deteriorating the operation conditions in case of high discrepancies between forecasted and actual values.

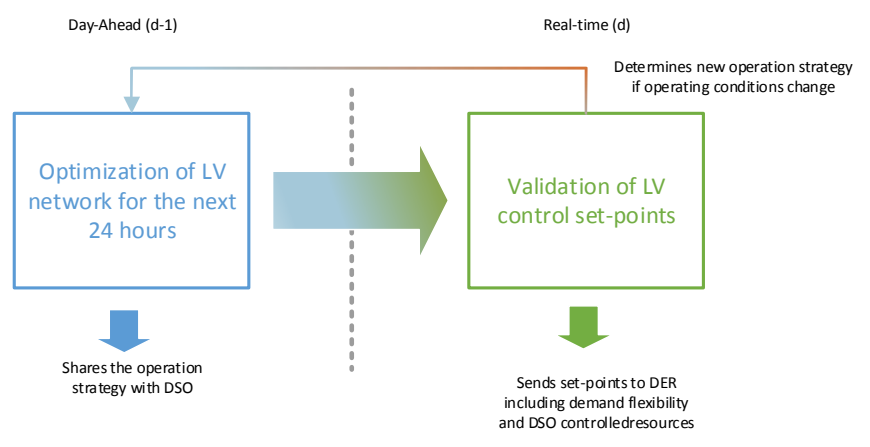

Figure 2. General Architecture of LV Network Optimization Tool

\section{RESULTS}

The proposed tool was tested in a real Portuguese LV distribution network. In this network, it was assumed that two storage units are included: one storage unit located at the 
secondary substation and one storage unit connected to a feeder. The storage unit located at secondary substation is assumed to be a $50 \mathrm{~kW} / 47 \mathrm{kWh}$ battery considering a $90 \%$ efficiency for both charge and discharge. The storage unit located at the feeder is assumed to be a $30 \mathrm{~kW} / 30 \mathrm{kWh}$ battery considering an efficiency of $90 \%$ for charge and discharge. The one-line diagram of the LV grid is shown in Figure 3.

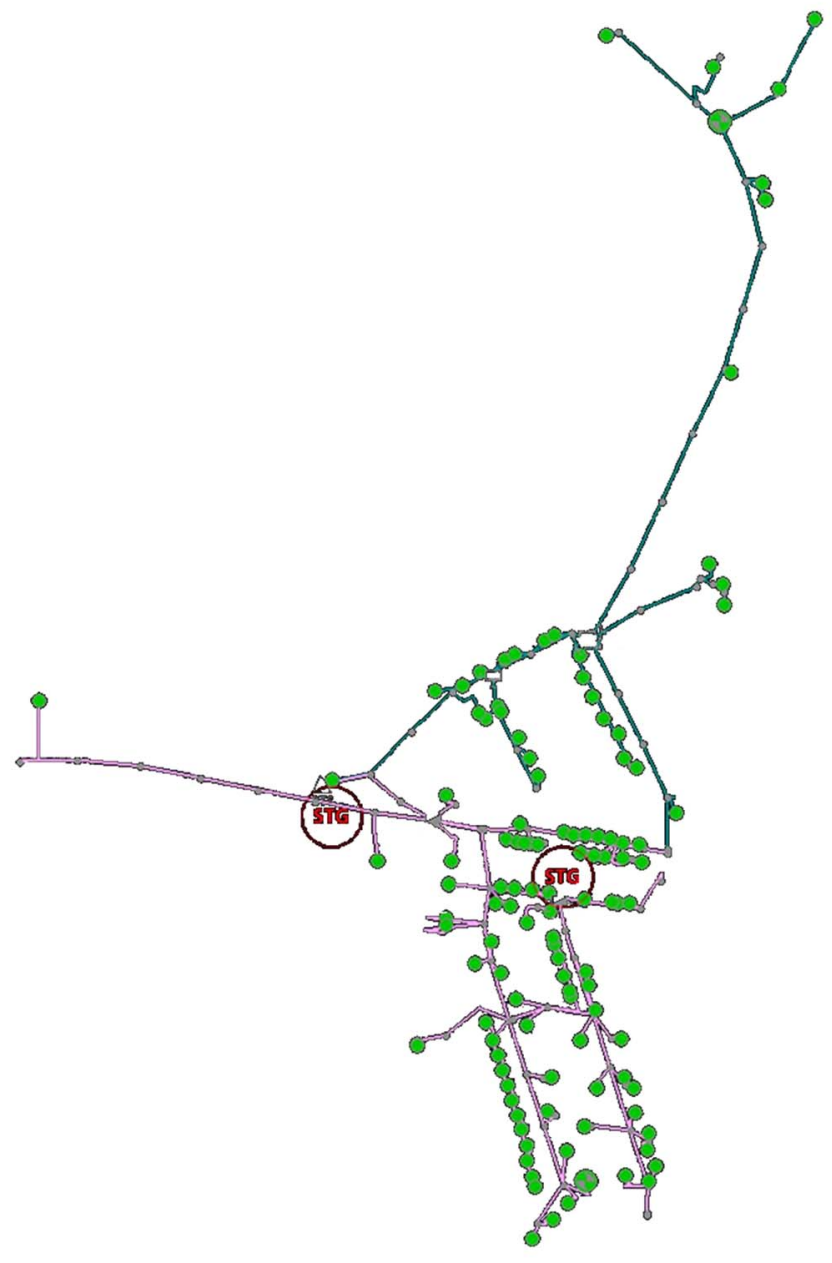

Figure 3. LV Test Network

\section{A. Scenario A}

The first scenario of operation considers a total load of 29.8 $\mathrm{kW}$ with $15.9 \mathrm{~kW}$ of PV generation. In Figure 4. the power management strategy is presented by running the multitemporal OPF for the next 24 hours, thus consisting of a set of 24 set-points (one set-point for each hour) for both active and for reactive power for the storage device in the feeder (which is the single control variable in this case). As can be observed, initially the battery charges during the first hour in order to ensure a minimum $S O C$ that was imposed.
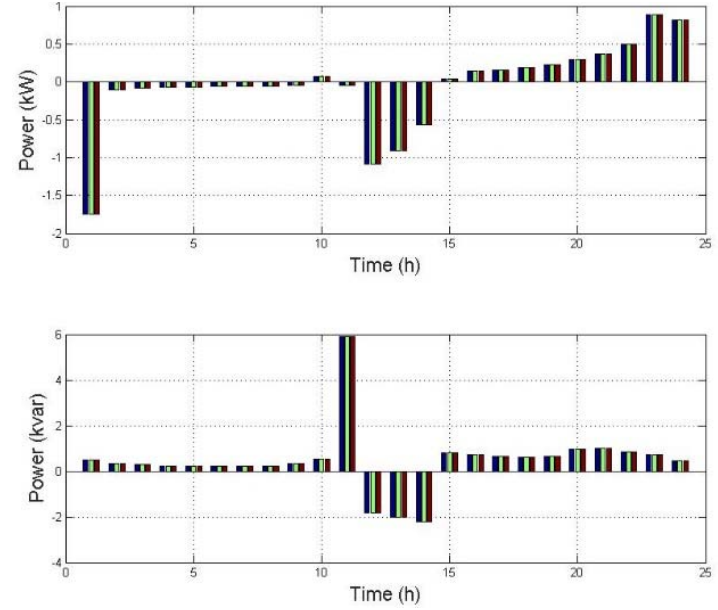

Figure 4. Active and Reactive Power Set-points for LV Storage Unit (Scenario A)

The Figure 5. and Figure 6. show the maximum, average and minimum voltage magnitudes in each phase obtained for all the network nodes along the day, when storage is not considered (Figure 5. ) and when the storage unit power management strategy is implemented (Figure 6.).
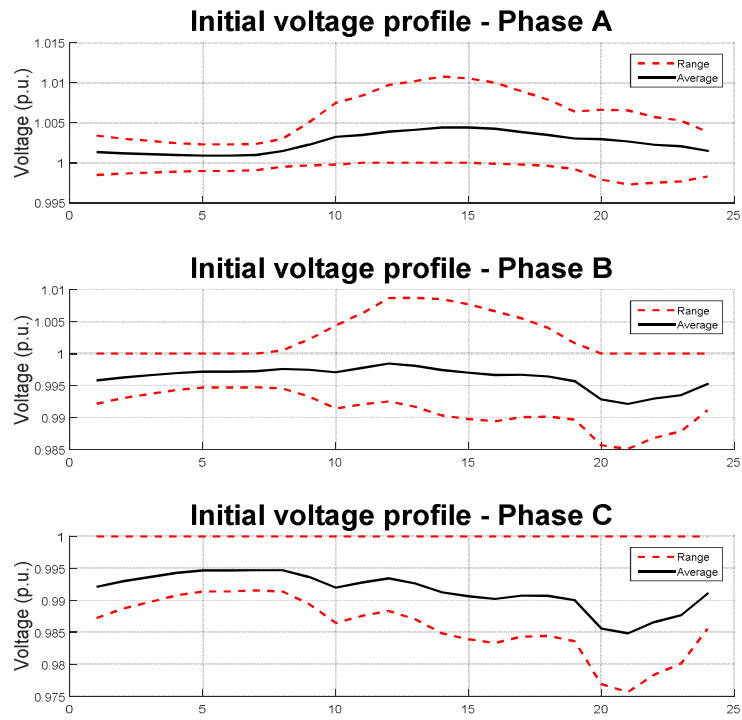

Figure 5. Voltage Magnitudes in Network Nodes without the Storage Unit (Scenario A)

The results show that voltage magnitudes remain within the limits defined of [0.95 - 1.05] p.u. along all the day. However, and although the main objective is not voltage profile improvement, the power management strategy defined by the multi-temporal OPF causes an overall improvement in the voltage profile in all three phases. This is due to the fact that the storage device present is a three-phase unit with the same control for all the phases, therefore it injects the same amount of active / reactive power in each of the three phases. 


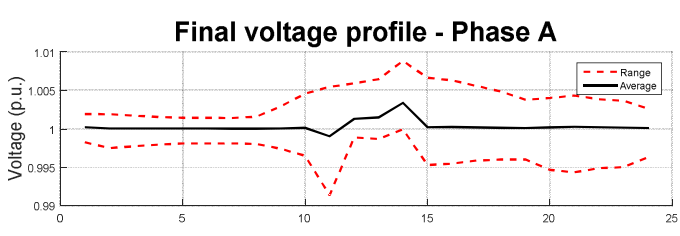

Final voltage profile - Phase B

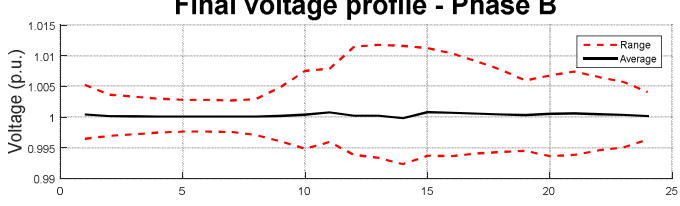

Final voltage profile - Phase C

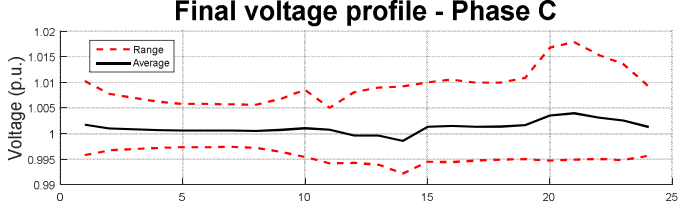

Figure 6. Voltage Magnitudes in Network Nodes with the Storage Unit (Scenario A)

\section{B. Scenario $B$}

In this scenario, an extreme condition of operation is considered with a total load of $74.4 \mathrm{~kW}$ and $10.6 \mathrm{~kW}$ of PV generation, to test the algorithm's efficiency facing a voltage violation condition. In this scenario, the demand flexibility is also used. It is considered $1.5 \mathrm{~kW}$ of maximum flexibility in the time period between 19:00 and 23:00 for each customer. The energy storage management strategy, for this scenario, is shown in Figure 7.
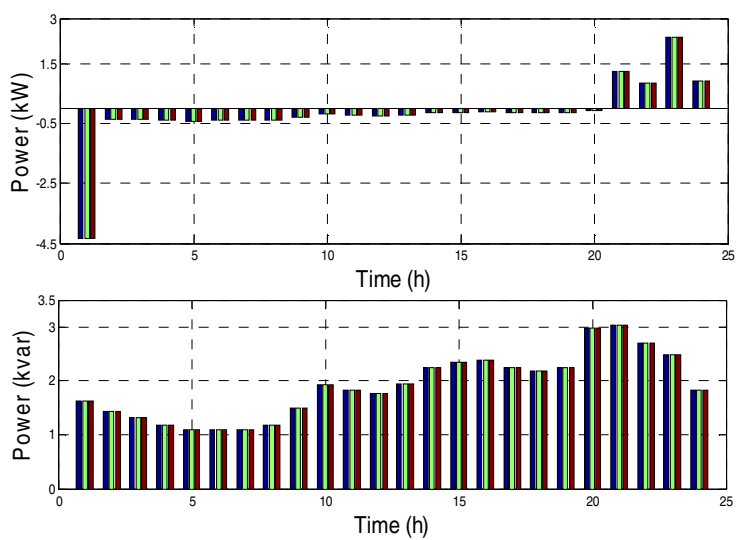

Figure 7. Active and Reactive Power Set-points for LV Storage Unit (Scenario B)

In Figure 8. , Figure 9. and in Figure 10. the active power set-points for the three flexible customers considered during the allowed time frame are presented. As previously discussed, it is considered that each one these selected consumers has a privately owned storage unit that, with a bilateral contact with the DSO, allows for these assets to participate in grid operation.

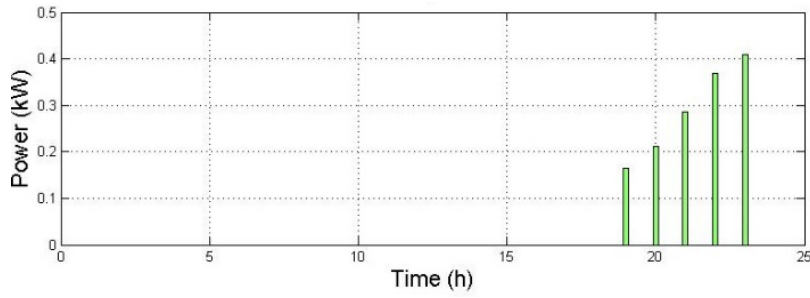

Figure 8. Active Power Set-points for costumer at bus 6 (Scenario B)

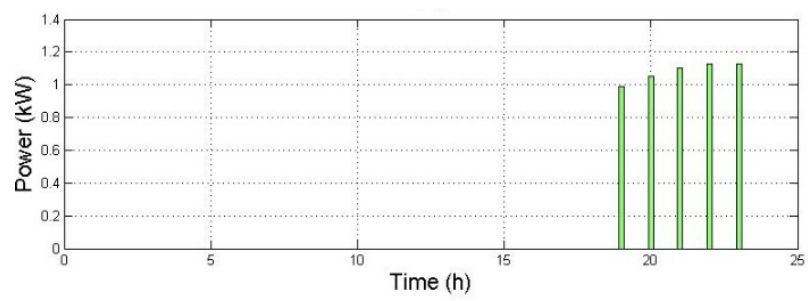

Figure 9. Active Power Set-points for costumer at bus 88 (Scenario B)

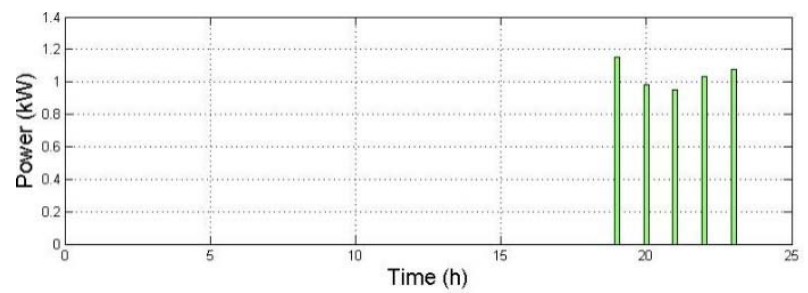

Figure 10. Active Power Set-points for costumer at bus 208 (Scenario B)

Figure 11. shows an undervoltage situation in phase $C$, corresponding to the period of time where the demand of power is higher $(19: 30-22: 00)$.
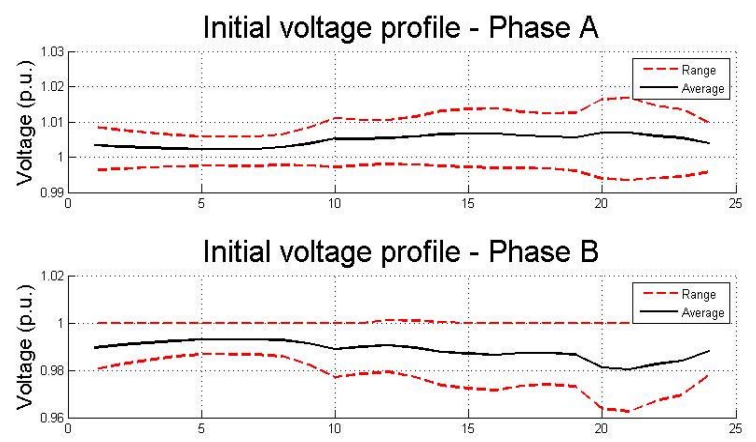

nitial voltage profile - Phase $\mathrm{C}$

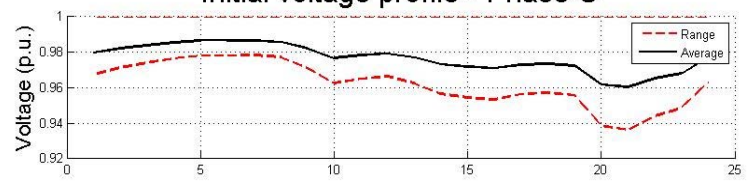

Figure 11. Voltage Magnitudes in Network Nodes with the Storage Unit and Demand Flexiblity (Scenario B)

Using demand flexibility and the storage unit, the network undervoltage occurrence was avoided, as shown in Figure 12. 

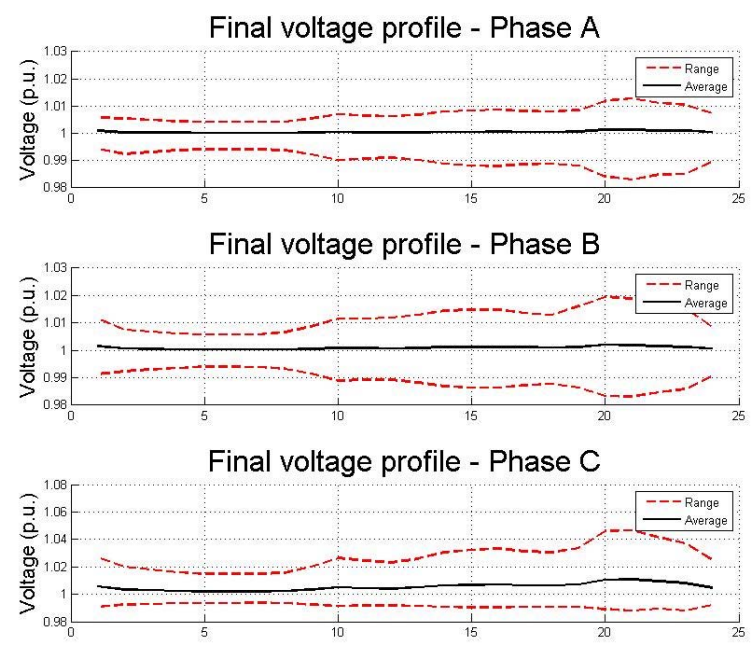

Figure 12. Voltage Magnitudes in Network Nodes with the Storage Unit and Demand Flexiblity (Scenario B)

\section{CONCLUSIONS}

From the DSO point of view, it is of the utmost importance to have distributed controllable resources in the LV network that can provide support to potential problems that may arise during operation. Allying these resources with the means to apply an optimized control enables expanding the DSO potential possibilities in terms of network operation.

Moreover, taking into consideration the existence of forecasts for mid-term operation exploration characteristics, it is possible to have a larger integration of RES without compromising the security of operation since these DER may cause a bidirectional power flows that may lead to operation problems that need to be properly managed. For these reasons, the importance of implementing means of controlling and coordinating the LV network assets will have increasing relevance in the DSO role. Providing solutions to manage the LV network regarding the macro objectives of the DSO will enable ensuring a safe and cost-effective operation.

The proposed algorithm is an example of such an optimization mechanism. It considers the available energy storages and demand flexibility as optimization control variables to determine the best suited operation profile given the time horizon considered. The input variables are the load and generation forecasts that depicts the state of the grid in that period. The optimization can consider different time horizons (i.e. 24 hours) and can be run in different instants (i.e. each hour) to update the solution regarding updated forecasts for the next hours.

The simulation obtained with the real network data provided has shown that the algorithm is reasonably fast (less than one minute) considering a machine equipped with am Intel Core i72600, $3.40 \mathrm{GHz}$ CPU and $8 \mathrm{~GB}$ of RAM memory. The variable with most significance in the simulation time delay is the time horizon considered. There is a linear correlation between the number of variables, the number of hours considered for optimization and the simulation time.

New optimization objectives and exploration scenarios can easily be adapted and simulated as the formulation is flexible enough. Still, based on the results obtained, the algorithm developed has proved that a LV operation optimization based of the network assets' coordination is possible and efficient with the formulation proposed.

\section{ACKNOWLEDGMENT}

The authors would like to thank the partners of the SENSIBLE project for the valuable discussions and, in particular, EDP NEW R\&D and EDP Distribuição for providing the network data for testing the developed algorithm.

\section{REFERENCES}

[1] J. A. P. Lopes, N. Hatziargyriou, J. Mutale, P. Djapic, and N. Jenkins, "Integrating distributed generation into electric power systems: A review of drivers, challenges and opportunities," Electr. Power Syst. Res., vol. 77, no. 9, pp. 1189-1203, Jul. 2007.

[2] K. Rahbar, J. Xu, and R. Zhang, "Real Time Energy Storage Management for Renewable Integration in Microgrid An OffLine Optimization Approach in on Smart Grid," IEEE Trans. Smart Grid, vol. 6 SRC-G, pp. 124-134, 2015.

[3] S. Lu, S. Repo, D. D. Giustina, F. A. C. Figuerola, A. Löf, and M. Pikkarainen, "Real-Time Low Voltage Network Monitoring-ICT Architecture and Field Test Experience," IEEE Transactions on Smart Grid, vol. 6, no. 4. pp. 2002-2012, 2015.

[4] J. L. Meirinhos, D. E. Rua, L. M. Carvalho, and A. G. Madureira, "Multi-temporal Optimal Power Flow for voltage control in MV networks using Distributed Energy Resources," Electr. Power Syst. Res., vol. 146, pp. 25-32, May 2017.

[5] M. Heleno et al., "Optimizing PV self-consumption through electric water heater modeling and scheduling," 2015 IEEE Eindhoven PowerTech.pp. 1-6, 2015.

[6] A. Madureira et al., "The Impact of Solar Power Forecast Errors on Voltage Control in Smart Distribution Grids," Proc. 23rd Int. Conf. Electr. Distrib. (CIRED 2015), Lyon, Fr., vol. 2015 SRC, pp. 1-5, 2015.

[7] V. H. Quintana, G. L. Torres, and J. Medina-Palomo, "Interior-point methods and their applications to power systems: a classification of publications and software codes," IEEE Transactions on Power Systems, vol. 15, no. 1. pp. 170-176, 2000.

[8] F. Capitanescu, M. Glavic, D. Ernst, and L. Wehenkel, "Interior-point based algorithms for the solution of optimal power flow problems," Electr. Power Syst. Res., vol. 77, no. 5-6, pp. 508-517, 2007.

[9] R. J. Vanderbei and D. F. Shanno, "An Interior-Point Algorithm for Nonconvex Nonlinear Programming," Comput. Optim. Appl., vol. 13, pp. 231-252, 1999.

[10] S. M. Souza, M. Gil, J. Sumaili, A. G. Madureira, and J. A. P. Lopes, "Operation scheduling of prosumer with renewable energy sources and storage devices," 2016 13th International Conference on the European Energy Market (EEM). pp. 1-5, 2016. 